\title{
Marriage predicts for survival in patients with stage III non-small-cell lung cancer
}

\author{
Melissa AL Vyfhuis, MD, PhD, ${ }^{\mathrm{a}}$ Josephine L Feliciano, MD, ${ }^{\mathrm{b}}$ Søren M Bentzen, $\mathrm{PhD}$, \\ DMSc, ${ }^{a}$ Martin J Edelman, MD, ${ }^{c}$ Katherine A Scilla, MD,${ }^{a}$ Neha Bhooshan, MD, PhD, ${ }^{a}$ \\ Whitney M Burrows, MD, ${ }^{a}$ Elizabeth M Nichols, MD, ${ }^{a}$ Mohan Suntharalingam, MD, \\ MBA, ${ }^{a}$ Steven J Feigenberg, MD, ${ }^{\mathrm{d}}$ and Pranshu Mohindra, $\mathrm{MD}^{\mathrm{a}}$
}

aUniversity of Maryland Marlene and Stewart Greenebaum Comprehensive Cancer Center, University of Maryland School of Medicine, Baltimore, Maryland; 'bidney Kimmel Comprehensive Cancer Center, Johns Hopkins School of Medicine, Baltimore, Maryland; ' Fox Chase Cancer Center, Philadelphia, Pennsylvania; and ${ }^{\mathrm{d} A b r a m s o n}$ Cancer Center, University of Pennsylvania School of Medicine, Philadelphia, Pennsylvania

Background Comprehensive analysis of prognostic significance of marital status in patients with stage III non-small-cell lung cancer (NSCLC) when adjusted for patient-, disease-, and treatment-specific factors, including the interaction with racial, nutritional, and immunologic status, is lacking.

Objective To evaluate whether marital status is an independent predictor of clinical outcomes in patients with stage III NSCLC who are treated uniformly with curative intent.

Methods The Kaplan-Meier method and Cox proportional hazards model were used to estimate the overall survival and freedom from recurrence (FFR) in 355 patients with stage III NSCLC who were treated during 2000-2013.

Results $52 \%$ of patients in the cohort were married and were more likely to self-identify as white $(P<.0001)$, reside in zip codes with a higher household median income $(P<.0001)$, have Eastern Cooperative Oncology Group Performance Status of $0(P=$ $.001)$, have higher pretreatment albumin $(P=.009)$, undergo surgery $(P=.001)$, and have insurance $(P=.029)$. On multivariate analysis, marital status remained an independent predictor of survival and was associated with a $40 \%$ decreased risk of death $(P$ $<.0001)$, further stratifying outcomes beyond gender and stage grouping. FFR was comparable between the 2 groups $(P=.108)$. Limitations Retrospective analysis; information on individual support system beyond the marital and insurance status and zip code income was not available.

Conclusions In a cancer such as NSCLC, in which modern therapeutic approaches have yielded only modest survival improvements despite considerable treatment-related toxicity, marital status remains an independent predictor for survival. Marriage is likely a surrogate for better psychosocial support; the scale of survival improvements seen justifies investments into supportive care interventional strategies to help advance overall outcomes.

Funding/sponsorship None

$\mathrm{N}$ on-small-cell lung cancer (NSCLC) remains the leading cause of cancer death in the United States, where $29 \%$ of patients will present with stage III disease., ${ }^{1,2}$ Ongoing research efforts seek to improve these outcomes using novel systemic therapy options or modern radiation techniques. However, there have also been recent studies showing the importance of marital and/or partner status on clinical outcomes..$^{3-7}$ For example, in a large Surveillance, Epidemiology, and End Results (SEER) analysis of 734,889 patients diagnosed with several types of cancer (including lung cancer), patients identified as married were less likely to present with metastatic disease, more likely to receive definitive therapy, and had superior cancer-related mortality even after adjusting for other variables such as cancer stage and treatment when compared with single patients. ${ }^{3}$ Population-based assessments are important in relaying information about trends and general outcomes based on marital status, but because they are large, they often lack patient-specific information such as nutrition, immunologic status, and variability in treatment paradigms, all of which can independently have an impact on overall survival (OS) in stage III NSCLC..$^{8-10}$ In addition, population analyses have typically included patients of all cancer stages and hence involved a multitude of treatment approaches ranging from curative to palliative. There are limited well-annotated institutional

Accepted for publication September 6, 2018. Correspondence: Melissa AL Vyfhuis, MD, PhD; mvyfhuis@umm.edu or Josephine L Feliciano, MD; jfelici4@jhmi.edu. Disclosures: The authors report no disclosures or conflicts of interest. JCSO 2018;16(5):e194-e201. @2018 Frontline Medical Communications. doi: https://doi.org/10.12788/jcso.0427 
data on the association of marital status on nonmetastatic, locally advanced (LA-NSCLC) in the setting of National Comprehensive Cancer Network-guided, standard-of-care definitive treatment.

The objective of this analysis is to evaluate the effect of marital status on OS and freedom from recurrence (FFR) in patients with stage III NSCLC who were treated at a National Cancer Institute-designated cancer center with curative intent from 2000 through 2013. We performed a detailed multivariate analysis (MVA) of patient-, disease-, and treatment-specific factors, including the interaction with racial, nutritional, and immunologic status, which to our knowledge has not been previously reported, to comprehensively evaluate the benefit of marital status in patients with LA-NSCLC.

\section{Methods}

\section{Patient population and treatment}

From January 2000 through December 2013, 355 patients diagnosed with clinical stage III NSCLC (American Joint Committee on Cancer 7th edition) were definitively treated at the University of Maryland in Baltimore, Maryland. Their clinical data were retrospectively analyzed under internal review board approval (GCC 1175, Thoracic Oncology Database). All of the patients were evaluated before treatment by a multidisciplinary team consisting of thoracic surgeons and medical and radiation oncologists. Before treatment, the patients underwent standard work-up, which included systemic imaging with positronemission (PET), computed-tomographic (CT), PET-CT, and/or bone scan, brain imaging consisting of magneticresonance imaging or CT with contrast, and routine blood. Patients had documentation of mediastinal disease by either imaging, mediastinoscopy, or endobronchial ultrasound biopsy.

Definitive therapy was administered using the backbone of chemoradiation therapy (CRT) with (trimodality) or without (bimodality) surgical resection. Concurrent CRT was typically administered with weekly carboplatin-paclitaxel (areas under the curve [AUCs], 2 and $50 \mathrm{mg} / \mathrm{m}^{2}$, respectively) and was generally followed with 2 cycles of consolidative treatment with definitive doses of carboplatin-paclitaxel (AUCs, 5-6 and 200-225 $\mathrm{mg} / \mathrm{m}^{2}$, respectively) as tolerated. The entire cohort was also assessed for possible trimodality therapy at the time of initial diagnosis, and patients who were potential surgical candidates were reassessed for mediastinal nodal clearance following repeat radiographic staging after fulldose CRT. Patients who experienced pathologic mediastinal clearance of disease underwent resection followed by consolidative chemotherapy. Unless there was evidence of disease progression, patients who did not have mediastinal lymph node clearance or who were found not to be a surgical candidate proceeded directly to consolidative chemotherapy. The details of patient selection for trimodality therapy and the oncological outcomes have been previously reported. ${ }^{10}$ For follow-up, patients were normally followed with serial CT or PET-CT scans as clinically indicated every 3 months for the first year, 4 to 6 months for the next 2 to 5 years, and then yearly thereafter.

For the analysis, patients were categorized as being either married or single based on self-reporting. As a surrogate for nutrition status, patients were stratified into 4 pretreatment body mass index (BMI) cohorts based on the following World Health Organization criteria: underweight, <18.5 $\mathrm{kg} / \mathrm{m}^{2}$; normal weight, 18.5 to $<25 \mathrm{~kg} / \mathrm{m}^{2}$; overweight, 25 to $<30 \mathrm{~kg} / \mathrm{m}^{2}$; and obesity, $\geq 30 \mathrm{~kg} / \mathrm{m}^{2}$. Pretreatment albumin was also evaluated as a continuous variable. For assessment of immunological status, neutrophil-to-lymphocyte ratio (NLR) was calculated at the time of diagnosis by dividing the absolute neutrophil count by the absolute lymphocyte count.

\section{Statistics}

We used the Pearson chi-square test to compare categorical variables. OS was calculated from the date of diagnosis (by biopsy of either primary tumor or mediastinal nodes) to the time of death or date of last follow-up. Patients were only censored if they were lost to follow-up. FFR was determined by the date of diagnosis to the time of first failure, with either distant or locoregional disease progression. For this analysis, patients were censored at the time of their last follow-up or death. The Kaplan-Meier product limit method was used to estimate OS and FFR, and we applied the log-rank test to compare outcomes between the 2 cohorts.

We conducted the multivariate analyses using Cox regression with forward model selection. Variables analyzed included age ( $<60$ vs $\geq 60$ years), sex, race (black vs nonblack), median household income, insurance status (Yes vs No), Eastern Cooperative Oncology Group Performance Status (ECOG PS) (range: 0-3; 0 = fully active and 3 = capable of limited self-care, confined to bed/chair $>50 \%$ of day) at time of diagnosis ( $0 \mathrm{vs} \geq 1$ ), pre-CRT BMI, smoking (pack-years), chronic obstructive pulmonary disorder (Yes vs No), Charlson Comorbidity Index score $(\leq 6$ vs > 7; range, 3-15; this score takes into consideration age, cardiovascular disease, malignancy, and other chronic conditions to calculate 1-year mortality), histology, calculated pretreatment NLR (as a continuous variable), pretreatment albumin (as a continuous variable), $\mathrm{T}$ stage, $\mathrm{N}$ stage, overall stage (IIIA vs IIIB), radiation technique (3D-CRT vs intensity-modulated radiation therapy [IMRT]), date of diagnosis (divided into quartiles based on proportion diagnosed by years: 2000-2002, 2003-2005, 2006-2009, 2010-2013), use of trimodality therapy, and consolidation chemotherapy. SPSS software (version 23.0) was used for statistical analysis (IBM Corp, Armonk, NY). 


\section{Original Report}

TABLE 1 Baseline patient, disease, and treatment characteristics ( $N=355$ )

Marital status

\begin{tabular}{|c|c|c|c|}
\hline Characteristic & $\begin{array}{l}\text { Married, } \\
\text { no. patients (\%) } \\
\text { (n = 185) }\end{array}$ & $\begin{array}{l}\text { Not married, } \\
\text { no. patients (\%) } \\
(\mathrm{n}=170)\end{array}$ & $P$-value \\
\hline \multicolumn{4}{|l|}{ Age, y } \\
\hline Median [range] & 61 [30-86] & 59.5 [38-84] & .960 \\
\hline$\geq 60$ & $100(54.1)$ & $86(51.2)$ & .590 \\
\hline \multicolumn{4}{|l|}{ Sex } \\
\hline Male & 113 (55.7) & $90(53.9)$ & .122 \\
\hline \multicolumn{4}{|l|}{ Race } \\
\hline White & $126(68.1)$ & $72(42.4)$ & \\
\hline Black & $53(28.6)$ & $97(57.1)$ & $<.0001$ \\
\hline Other & $6(3.2)$ & $1(0.5)$ & \\
\hline
\end{tabular}

Above median income ${ }^{a}$

\begin{tabular}{|c|c|c|c|}
\hline$\geq \$ 43,723$ & $117(63.6)$ & 55 (33.3) & $<.0001$ \\
\hline \multicolumn{4}{|c|}{ nsurance status } \\
\hline Yes & $161(87)$ & $137(80.6)$ & \multirow{3}{*}{.029} \\
\hline No & $16(8.6)$ & $28(16.5)$ & \\
\hline Unknown & $8(4.4)$ & $5(2.9)$ & \\
\hline \multicolumn{4}{|c|}{ ECOG Performance Status ${ }^{b}$} \\
\hline 0 & $102(55.1)$ & $63(37)$ & \multirow{3}{*}{.001} \\
\hline$\geq 1$ & $81(43.8)$ & $105(61.8)$ & \\
\hline Unknown & $2(1.1)$ & $2(1.2)$ & \\
\hline
\end{tabular}

Pretreatment $\mathrm{BM} \mathrm{K}^{\mathrm{c}}$

Median, $\mathrm{kg} / \mathrm{m}^{2}$ [range]

26.3 [16.1-41.3]

$24.4[11.2-43.9]$

.050

Obese

42 (22.7)

$35(20.6)$

Overweight

53 (28.6)

Normal

49 (26.5)

34 (20)

Underweight

7 (3.8)

58 (34.1)

.095

Unknown

$34(18.4)$

12 (7.1)

31 (18.2)

Smoking, pack-years

Median [range]

40 [0-180]

40 [0-212]

.818

COPD diagnosis

$\begin{array}{llll}\text { Yes } & 49(27) & 51(30) & .477\end{array}$

Charlson Comorbidity Index score $^{\text {d }}$

$\begin{array}{lcc}\leq 6 & 99(53.5) & 90(52.9) \\ >7 & 86(46.5) & 79(46.5) \\ \text { Unknown } & - & 1(0.6) \\ \text { Histology } & 61(33) & 52(30.6) \\ \text { Adenocarcinoma } & 48(25.9) & 56(32.9) \\ \text { Squamous cell } & 62(33.5) & 50(29.4) \\ \text { NSCLC (NOS) } & 14(7.6) & 12(7.1) \\ \text { Other } & & \end{array}$

Other 
Cntinued from on previous page

\begin{tabular}{|c|c|c|c|}
\hline \multirow[b]{2}{*}{ Characteristic } & \multicolumn{2}{|c|}{ Marital status } & \multirow[b]{2}{*}{$P$-value } \\
\hline & $\begin{array}{l}\text { Married, } \\
\text { no. patients (\%) } \\
\text { (n= 185) }\end{array}$ & $\begin{array}{l}\text { Not married, } \\
\text { no. patients (\%) } \\
(n=170)\end{array}$ & \\
\hline \multicolumn{4}{|l|}{ Pretreatment NLR } \\
\hline Median [range] & $3.22[0.22-57.9]$ & $3.30[0.67-37.5]$ & .393 \\
\hline \multicolumn{4}{|c|}{ Pretreatment albumin, $\mathrm{g} / \mathrm{dL}$} \\
\hline Median [range] & 3.7 [0.80-5.0] & $3.6[0.70-4.9]$ & .009 \\
\hline \multicolumn{4}{|l|}{ T stage ${ }^{e f}$} \\
\hline $\mathrm{TX}$ & $10(5.4)$ & $8(4.7)$ & \\
\hline$\leq \mathrm{T} 2$ & $90(48.9)$ & $71(42)$ & .361 \\
\hline$\geq \mathrm{T} 3$ & $84(45.7)$ & $90(53.3)$ & \\
\hline
\end{tabular}

\begin{tabular}{|c|c|c|c|}
\hline \multicolumn{4}{|l|}{$N$ stage $^{\text {eg }}$} \\
\hline$N X$ & $2(1.1)$ & $1(0.6)$ & \multirow{4}{*}{.906} \\
\hline$\leq \mathrm{N} 1$ & $31(16.8)$ & $25(14.8)$ & \\
\hline N2 & $112(60.9)$ & $106(62.7)$ & \\
\hline N3 & $39(21.2)$ & $37(21.9)$ & \\
\hline \multicolumn{4}{|l|}{ Overall stage } \\
\hline IIIA & 109 (58.9) & $91(53.5)$ & \multirow[t]{2}{*}{.306} \\
\hline IIIB & $76(41.1)$ & $79(46.5)$ & \\
\hline \multicolumn{4}{|l|}{ Treatment } \\
\hline Trimodality & $59(31.9)$ & $29(17.1)$ & \multirow[t]{2}{*}{.001} \\
\hline Bimodality & $126(68.1)$ & 141 (82.9) & \\
\hline \multicolumn{4}{|c|}{ Type of chemoradiation } \\
\hline Concurrent & $175(94.6)$ & $152(89.4)$ & \multirow[t]{2}{*}{.070} \\
\hline Sequential & $10(5.4)$ & $18(10.6)$ & \\
\hline \multicolumn{4}{|c|}{ Radiation dose delivered, $\mathrm{Gy}^{h}$} \\
\hline Median [range] & $64.8[10.8-70.2]$ & $63[19.8-81.6]$ & .126 \\
\hline$\geq 60$ & 154 (93.9) & $139(88)$ & .063 \\
\hline \multicolumn{4}{|c|}{ Radiation technique } \\
\hline 3D-confromal & $132(77.6)$ & $103(69.1)$ & \multirow[t]{2}{*}{.098} \\
\hline IMRT & $38(22.4)$ & $46(30.9)$ & \\
\hline \multicolumn{4}{|c|}{ Adjuvant chemotherapyi } \\
\hline Yes & $121(73.3)$ & $91(65.5)$ & .137 \\
\hline
\end{tabular}

BMI, body mass index; COPD, chronic obstructive pulmonary disease; ECOG, Eastern Cooperative Oncology Group; IMRT, intensity-modulated radiation therapy; NLR, neutrophil-to-lymphocyte ratio; NOS, not otherwise specified; NSCLC, non-small cell lung cancer

aData not available for 6 patients. ECOG PS range, $0-3$, with $0=$ fully active and $3=$ capable of limited self-care, spending $>50 \%$ of the day in a chair or in bed. 'BMI, normal, $18.5-25 \mathrm{~kg} / \mathrm{m}^{2}$. ${ }^{\mathrm{d}}$ Charlson Comorbidity Index score range, $3-15$ (this score takes into consideration age, cardiovascular disease, malignancy, and other chronic conditions to calculate 1 -year mortality). ${ }^{\mathrm{e} T}$ and $\mathrm{N}$ staging not available for 2 patients. $\mathrm{T}$ T staging is reflected of $\mathrm{AJCC} 7$ th edition. $9 \mathrm{~N}$ staging is reflected of AJCC 7th edition. 'Data not available for 33 patients. 'Data not available for 36 patients. iData not available for 8 patients.

\section{Results}

\section{Treatment cohorts}

Table 1 compares and summarizes patient demographics, disease, and treatment characteristics for married (n $=185 ; 52.1 \%)$ and nonmarried $(\mathrm{n}=170 ; 47.9 \%)$ patients.
Married patients were more likely to self-identify as being white $(P<.0001)$, reside in zip codes with a higher household median income $(P<.0001)$, have an ECOG PS of 0 $(P=.001)$, have a higher distribution of pretreatment albumin levels $(P=.009)$, and undergo trimodality therapy 
$(P=.001)$, and they were twice as likely to have insurance $(P=.029)$. Both cohorts were evenly distributed in terms of $\mathrm{T}$ stage, $\mathrm{N}$ stage, and overall staging. There was no difference in pretreatment NLR or pretreatment BMI between married and single patients. Concurrent CRT was used in more than $85 \%$ of patients in both groups, with approximately two-thirds also receiving consolidation chemotherapy (Table 1). Median delivered radiation dose was 64.8 Gy (range, 10.8-81.6 Gy). There was no statistically significant difference in radiation dose delivered to either group, with nearly $90 \%$ of the cohort receiving $\geq 60 \mathrm{~Gy}$.

\section{OS and FFR}

With a median follow-up of 15 months for all patients and 89 months for surviving patients (range, 1-184 months), married patients had improved OS when compared with the single cohort, with a median survival of 29.6 and 18.4 months, respectively (unadjusted hazard ratio [HR] of married vs nonmarried, .640; 95\% confidence interval [CI], 0.502-0.816; $P<.0001$; Figure 1A). The estimated $2-$ and 5 -year OS for married and single patients were $56 \%$ and $31 \%$ and $38.6 \%$ and $15 \%$, respectively. When stratified by stage, married patients with stage IIIB disease (median survival, 25 months; Figure 1B) had a similar survival to unmarried patients with stage IIIA disease (median survival, 24 months; Figure 1B). In stage IIIA patients, marital status was associated with an unadjusted HR of .696 (95\% CI, 0.497-0.974; $P=.035)$, with a larger OS benefit seen in the IIIB group (unadjusted HR, .601; 95\% CI, 0.422$0.856 ; P=.005)$.

Survival as it pertains to marital status was further stratified by sex (Figure 2A) and race (Figure 2B). Married men had an improved estimated median survival of 30 months when compared with single men, whose median survival was 16 months (unadjusted HR, .541; 95\% CI, 0.3920.746; $P<.0001)$. On the other hand, marital status had no statistically significant effect on OS when comparing married women with their single counterparts (unadjusted HR, .717; 95\% CI, 0.491-1.048; $P=.085$; Figure $2 \mathrm{~A}$ ), with an overall median survival of approximately 28 months for the entire female cohort. Stratification by race also showed similar results, with married nonblack patients demonstrating better OS when compared with single nonblack patients (HR, .586; 95\% CI, 0.420-0.820; $P=.002$; Figure 2B), with a median survival of 29 and 17 months, respectively. Black patients also had a similar improvement in survival when comparing the married (median survival, 30 months) and nonmarried groups (median survival, 19.6 months; unadjusted HR, .676; 95\% CI, 0.457-1.000; $P=.050 ;$ Figure 2B).

FFR did not differ between the 2 groups, with a median time to failure of 17 and 15 months for married and nonmarried patients, respectively (unadjusted HR, .799; 95\% CI, 0.607-1.051; $P=.108$; Figure 3). Estimated 2- and 5-year

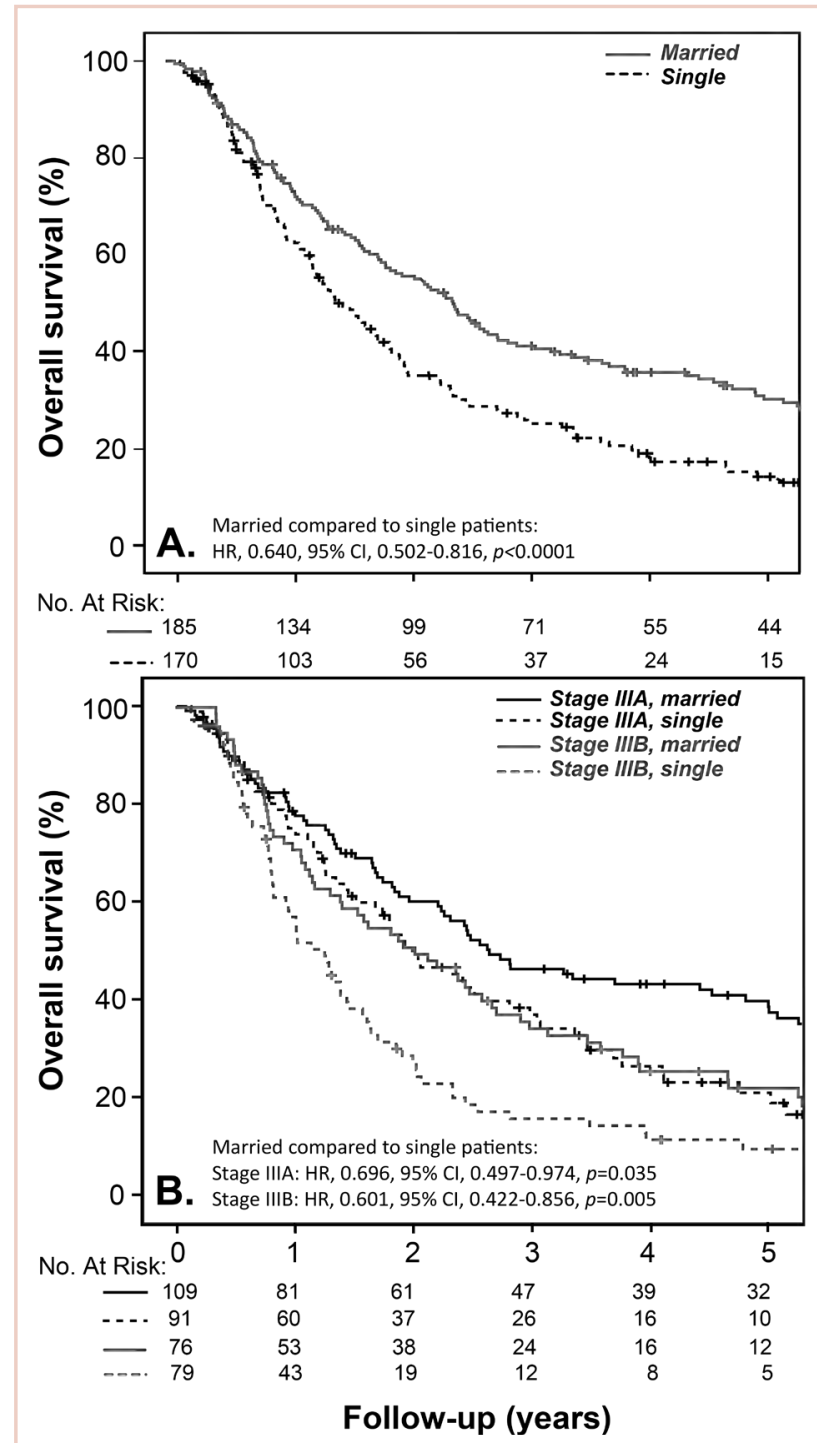

FIGURE 1 Kaplan-Meier plot of overall survival of stage III NSCLC patients based on $\mathbf{A}$, marital status and $\mathbf{B}$, marital status stratified by overall stage. Unadjusted hazard ratios are reported in each figure.

FFR for married and nonmarried patients were $39.4 \%$ and $27 \%$ and $31.5 \%$ and $18.5 \%$, respectively (Figure 3 ).

\section{Clinical predictors of survival}

On MVA, factors that were independent predictors for OS are summarized in Table 2. Risk of death was reduced by approximately $65 \%$ and $45 \%$ in patients who underwent trimodality treatment $(P<.0001)$ or were able to undergo consolidative chemotherapy $(P=.004)$ when compared with those who were treated definitively with bimodality treatment or did not undergo systemic doses of adjuvant chemotherapy, respectively. Having insurance $(P=.048)$ and use of IMRT over 3D-CRT $(P=.008)$ was 


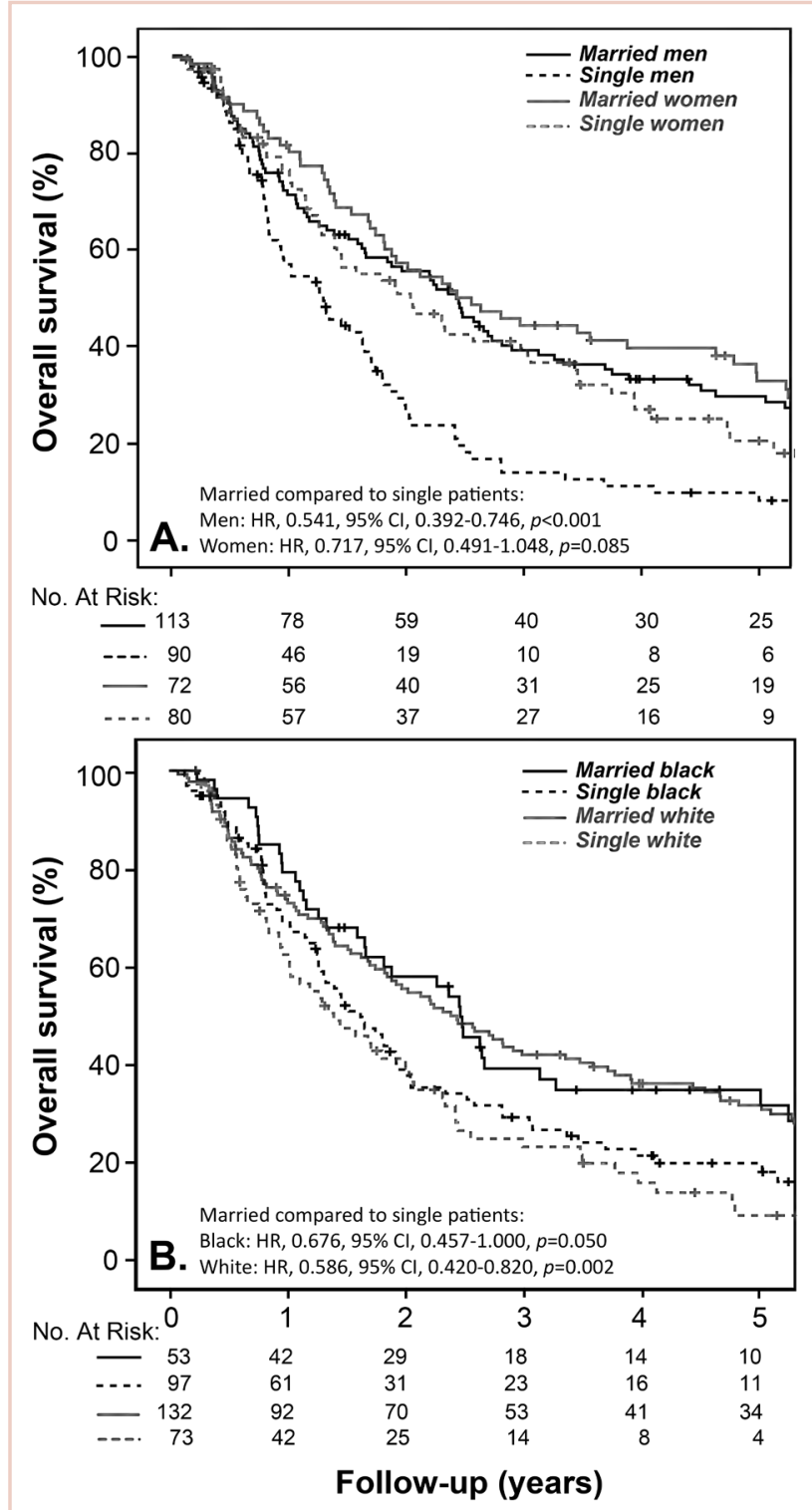

FIGURE 2 Kaplan-Meier plot of overall survival of stage III NSCLC patients based on $\mathbf{A}$, marital status stratified by sex or B, marital status stratified by race. Unadjusted hazard ratios are reported in each figure.

associated with a reduction of mortality by about half in this cohort. Both gender (improved OS with female sex; $P=.004$ ) and marital status (improved OS with marriage; $P=.006)$ were associated with a decreased the risk of death by $40 \%$ (Table 2 ). By contrast, a higher NLR resulted in a 1.1-times increased risk of death $(P=.001)$.

\section{Discussion}

Our study continues to support the notion that marital status is an independent indicator of survival in stage III NSCLC (adjusted HR, .59; 95\% CI, 0.404-0.859;

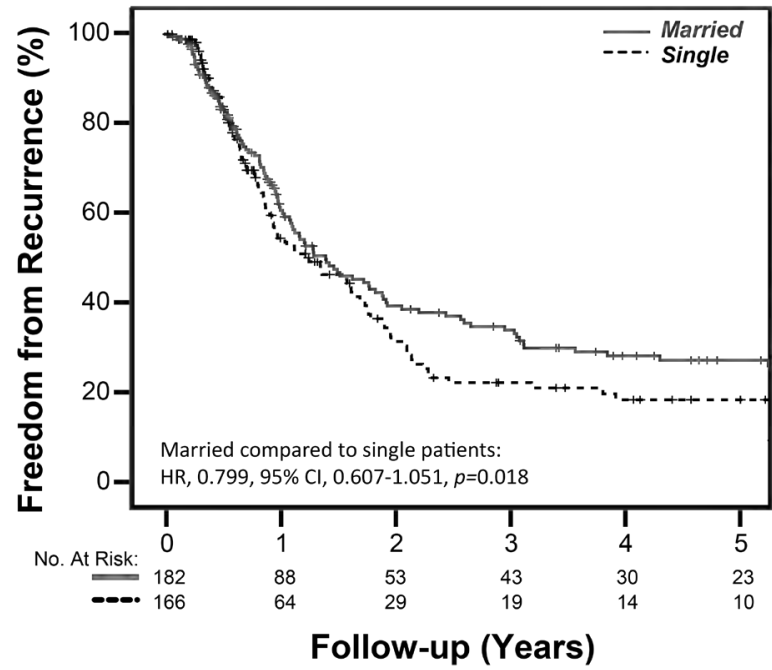

FIGURE 3 Kaplan-Meier plot of freedom from recurrence of stage III NSCLC stratified by marital status. Unadjusted hazard ratios are reported.

$P=.006)$. The benefit of marriage in this population seems to be better than that reported in the SEER analysis for all stages, wherein the HR for death of married patients compared with their single counterparts was .85 (95\% CI, $0.83-0.87)$. In their analysis, the investigators hypothesized that this survival advantage could partially be explained by better access to health care and adherence to therapy, as was supported by the higher likelihood of married patients presenting with localized disease and receiving definitive treatment. ${ }^{3}$ Another population-based study using the Florida Cancer Data System identified 161,228 lung cancer patients (NSCLC and small-cell lung histology included), and on MVA, marital status remained an important prognostic indicator for OS when compared with never-married patients $(\mathrm{HR}, .86 ; P=.001) .{ }^{6}$ In addition to typically including patients with all stages of diseases, population-based studies often include patients who receive a heterogeneous combination of treatment modalities, possibly confounding the analysis. Furthermore, large population analyses typically do not report on patient-specific variables such as nutrition (ie, BMI and albumin) or immunologic status (ie, NLR), both of which have been shown to be independent predictors of survival in LA-NSCLC. ${ }^{8,9}$

In contrast, some other studies have failed to demonstrate an OS advantage with marital status in patients with NSCLC. For example, in a meta-analysis that evaluated the influence of race, gender, and marital status on 1,365 nonoperative NSCLC patients who were enrolled in 9 Radiation Therapy Oncology Group (RTOG) trials, the investigators did not find marital status to be independently predictive of survival. ${ }^{11}$ In addition, for the 5,898 patients who were prospectively enrolled in a Mayo Clinic 
Lung Cancer Cohort (MCLCC), marital status was also found not to be prognostic for NSCLC outcomes when all stages of the disease were analyzed together. ${ }^{4}$ There are some possible confounding factors in these studies. Patients recruited for clinical trials tend to be healthier with a better performance status and have a support system (including close monitoring by the study team) when compared with the general population diagnosed with lung cancer. About $70 \%$ to $76 \%$ of the patients in both the RTOG and MCLCC studies were married, which is significantly higher than both the national average (51\%) and our group (52.1\%). Like other population-based studies, the MCLCC included patients with all stages getting a variety of treatments. Although no overall impact on survival was noted, the investigators noted that single, divorced, and widowed patients were more likely to not receive cancer therapy $(P<.0001)$. The marital status also influenced the choice of therapy, with subgroup analysis revealing inferior outcomes in widowed and divorced patients with stage IA, IIB, or IIIB disease. The authors also recognized an inherent referral bias from patients, with support system being typically seen at the Mayo clinics, which may have played an additional role. All of the patients in our analysis were appropriately staged and received curative-intent treatment by a team of physicians using essentially identical therapeutic strategies, thus minimizing some of these confounding factors. This allowed us to explore the impact of marital status while a patient was undergoing stage-appropriate treatment. We demonstrated a strong association with marital status and survival that even overcame the effects of stage (IIIA vs IIIB) on clinical outcomes (Figure 1B).

Furthermore, our analysis allowed us to explore the interaction of race and marital status more definitively because the demographics of the patients in the RTOG and MCLCC included 14\% and less than 3\% of patients identified as being nonwhite, respectively, in contrast to our analysis in which $41 \%$ of the patients self-identified as black. ${ }^{12}$ In our black population, marital status was associated with an observable improvement in OS, similar to our nonblack, pre- dominantly white (97\%) cohort (Figure 2B). Also, the results of our analysis may be a more accurate representation of the general population living in large urban or semiurban settings and further implies that an intact social support system could have a greater influence on clinical outcomes.

The current analysis is unique when compared with previous published studies in that beyond conventional demographic and treatment-related factors, we have comprehensively explored potential mechanisms that may explain the survival advantage seen in married patients by evaluating additional factors, such as functional status (ECOG and Charlson's scores), nutritional status (BMI and albu$\mathrm{min}$ ), immunologic characteristics (NLR), and other social factors (race, income, insurance status). Although married patients were more likely to have a higher BMI and albumin at diagnosis, when controlling for these factors in the multivariable analysis, marital status remained strongly prognostic (Table 2), suggesting that nutrition alone does not fully account for the observed survival advantage demonstrated. A similar conclusion can be drawn about immunologic status. NLR has previously been shown to be prognostic in a number of cancers, ${ }^{13-16}$ including in our own cohort. ${ }^{8}$ Although immune status remains an important predictor for OS in our locally advanced NSCLC population, when we take NLR into consideration in our analysis, marital status continues to be a strong indicator for survival (Table 2). In terms of other variables analyzed, insurance status was a significant predictor of OS in the MVA, though functional status and other social factors including race were not significant.

We also explored cancer control outcomes in the form of FFR. Married patients had an observable, although not statistically significant, improvement in FFR when compared with the single cohort (Figure 2). In our study, married patients were more likely to undergo trimodality therapy (Table 1), which has likely translated to the improvement of FFR seen in our group. In this case, marriage may serve as a surrogate for availability of a support system to undergo aggressive, potentially toxic treatment. ${ }^{3,17,18}$ Even in the setting of bimodality therapy, the RTOG 0617 study noted

\begin{tabular}{|c|c|c|c|}
\hline Characteristic & Hazard ratio & $95 \%$ confidence interval & $P$-value \\
\hline Trimodality & .440 & $0.292-0.662$ & $<.0001$ \\
\hline Insurance status & .491 & $0.243-0.994$ & .048 \\
\hline Radiation technique & .502 & $0.302-0.837$ & .008 \\
\hline Consolidation chemotherapy & .560 & $0.380-0.827$ & .004 \\
\hline Marital status & .590 & $0.404-0.859$ & .006 \\
\hline Sex & .598 & $0.421-0.849$ & .004 \\
\hline NLR & 1.051 & $1.020-1.082$ & .001 \\
\hline
\end{tabular}


about $17.5 \%$ treatment interruptions because of adverse effects or illness, with more than $30 \%$ of patients experiencing grade 3 or more esophagitis, irrespective of radiation technique. ${ }^{19}$ In these scenarios, in addition to receiving better attention to nutrition and care, significant others often provide emotional and social support that, in turn, can lead to better compliance. Social supports and sociodemographic factors are especially critical in patient populations in which access to health care is challenging.

Despite the compelling outcomes presented, our study suffers from the common limitations of retrospective analyses. Marital status, in this setting, most likely correlates with improved socioeconomic status and greater support, which have resulted in improved survival. Furthermore, although patients were self-classified as married or single, our data were not able to capture whether patients were single but lived with another adult or had other types of social support. However, even if there was a proportion of the unmarried cohort that had an alternate support system, separating them out is likely to further expand the differences. Quantifying the amount of social, emotional, or even spiritual support was not possible to accomplish in our analysis, though we know that all 3 can play a role in cancer outcomes. ${ }^{20,21}$ Further prospective studies would

\section{References}

1. Siegel RL, Miller KD, Jemal A. Cancer statistics, 2018. CA Cancer J Clin. 2018;68(1):7-30.

2. Goldstraw P, Chansky K, Crowley J, et al. The IASLC lung cancer staging project: proposals for revision of the TNM stage groupings in the forthcoming (eighth) edition of the TNM classification for lung cancer. J Thorac Oncol. 2016;11(1):39-51.

3. Aizer AA, Chen M-H, McCarthy EP, et al. Marital status and survival in patients with cancer. J Clin Oncol. 2013;31(31):3869-3876

4. Jatoi A, Novotny P, Cassivi S, et al. Does marital status impact survival and quality of life in patients with non-small cell lung cancer? Observations from the mayo clinic lung cancer cohort. Oncologist. 2007;12(12):1456-1463.

5. Kravdal H, Syse A. Changes over time in the effect of marital status on cancer survival. BMC Public Health. 2011;11:804.

6. Tannenbaum SL, Zhao W, Koru-Sengul T, Miao F, Lee D, Byrne MM. Marital status and its effect on lung cancer survival. Springerplus. 2013;2:504.

7. Ellis L, Canchola AJ, Spiegel D, Ladabaum U, Haile R, Gomez SL. Racial and ethnic disparities in cancer survival: the contribution of tumor, sociodemographic, institutional, and neighborhood characteristics [published online October 16, 2017]. J Clin Oncol. 2018;36(1):25-33.

8. Scilla KA, Bentzen SM, Lam VK, et al. Neutrophil-lymphocyte ratio is a prognostic marker in patients with locally advanced (stage IIIA and IIIB) non-small cell lung cancer treated with combined modality therapy. Oncologist. 2017;22(6):737-742.

9. Lam VK, Bentzen SM, Mohindra P, et al. Obesity is associated with long-term improved survival in definitively treated locally advanced non-small cell lung cancer (NSCLC). Lung Cancer. 2017;104:52-57.

10. Vyfhuis MAL, Bhooshan N, Burrows WM, et al. Oncological outcomes from trimodality therapy receiving definitive doses of neoadjuvant chemoradiation ( $\geq 60 \mathrm{~Gy}$ ) and factors influencing consideration for surgery in stage III non-small cell lung cancer. Adv Radiat Oncol. 2017;2(3):259-269.

11. Siddiqui F, Bae K, Langer CJ, et al. The influence of gender, race, and marital status on survival in lung cancer patients: analysis of radiation have to be done to completely understand how marital status can influence clinical decisions. Understanding whether marital status is a proxy for social provisions may help to identify populations at risk for inferior outcomes. These atrisk patients may benefit from targeted clinical interventions, such as closer physician follow-up, more aggressive supportive care, access to support groups, or nurse navigator visits.

\section{Conclusions}

In patients with locally advanced NSCLC treated with curative-intent following uniform treatment algorithms, marital status was linked with improvement in survival even when adjusted for other key variables, with the second highest HR (after insurance status) among pretreatment demographic variables. Although marriage is an unmodifiable factor in itself, it is most likely a surrogate for better psychosocial support. The scale of these positive survival improvements emphasizes the need to institute targeted supportive care strategies to help advance overall outcomes in a tumor for which modern therapeutic approaches (novel systemic therapy and radiation) have yielded only modest improvement in outcomes yet come at the cost of considerable treatment-related toxicity.

therapy oncology group trials. J Thorac Oncol. 2010;5(5):631-639.

12. Vyfhuis MAL, Bhooshan N, Molitoris J, et al. Clinical outcomes of black vs. non-black patients with locally advanced non-small cell lung cancer. Lung Cancer. 2017;114:44-49.

13. Beltran BE, Castro D, De La Cruz-Vargas JA, et al. The neutrophillymphocyte ratio is prognostic in patients with early stage aggressive peripheral T cell lymphoma [published online February 26, 2018]. Br J Haematol. doi:10.1111/bjh.15141.

14. Lee BM, Chung SY, Chang JS, Lee KJ, Seong J. The neutrophil-lymphocyte ratio and platelet-lymphocyte ratio are prognostic factors in patients with locally advanced pancreatic cancer treated with chemoradiotherapy. Gut Liver. 2018;12(3):342-352.

15. Najjar M, Agrawal S, Emond JC, Halazun KJ. Pretreatment neutrophil-lymphocyte ratio: useful prognostic biomarker in hepatocellular carcinoma. J Hepatocell Carcinoma. 2018;5:17-28.

16. Hu W, Yu J, Huang Y, Hu F, Zhang X, Wang Y. Lymphocyterelated inflammation and immune-based scores predict prognosis of chordoma patients after radical resection. Transl Oncol. 2018;11(2):444-449.

17. Mahal BA, Cooperberg MR, Aizer AA, et al. Who bears the greatest burden of aggressive treatment of indolent prostate cancer? Am J Med. 2015;128(6):609-616.

18. Inverso G, Mahal BA, Aizer AA, Donoff RB, Chau NG, Haddad RI. Marital status and head and neck cancer outcomes. Cancer. 2015;121(8):1273-1278.

19. Chun SG, Hu C, Choy H, et al. Impact of intensity-modulated radiation therapy technique for locally advanced non-small-cell lung cancer: a secondary analysis of the NRG oncology RTOG 0617 randomized clinical trial. J Clin Oncol. 2017;35(1):56-62.

20. Waite LJ, Lehrer EL. The benefits from marriage and religion in the United States: a comparative analysis. Popul Dev Rev. 2003;29(2):255-276

21. Osborne C, Ostir GV, Du X, Peek MK, Goodwin JS. The influence of marital status on the stage at diagnosis, treatment, and survival of older women with breast cancer. Breast Cancer Res Treat. 2005;93(1):41-47. 